\title{
Multidrug Resistant Gram-Negative Bacteria in Community-Acquired Pneumonia
}

\author{
Catia Cillóniz ${ }^{1,2,3,4}$, Cristina Dominedò ${ }^{5}$ and Antoni Torres ${ }^{1,2,3,4^{*}}$
}

\begin{abstract}
This article is one of ten reviews selected from the Annual Update in Intensive Care and Emergency Medicine 2019. Other selected articles can be found online at https://www.biomedcentral.com/collections/ annualupdate2019. Further information about the Annual Update in Intensive Care and Emergency Medicine is available from http://www.springer.com/ series/8901.
\end{abstract}

\section{Introduction: Why is this topic important?}

Community-acquired pneumonia (CAP) is associated with high morbidity and mortality worldwide [1]. Although several different bacteria and respiratory viruses can be responsible for CAP, Streptococcus pneumoniae (pneumococcus) remains the most common causative pathogen. A small proportion of CAP cases are caused by Gram-negative bacteria, especially Pseudomonas aeruginosa, Klebsiella pneumoniae, Acinetobacter baumannii and Stenotrophomona maltophilia $[2,3]$. The main problem concerning the treatment of Gram-negative bacterial infections is their related antibiotic resistance, reported as multidrug resistant $(\mathrm{MDR}=$ resistant to at least one agent in three or more groups of antibiotics), extensively drug resistant $(\mathrm{XDR}=$ resistant to at least one agent in all but two or fewer groups of antibiotics) and pan-drug resistant $(\mathrm{PDR}=$ resistant to all groups of antibiotics) [4]. This makes the clinical management of pneumonia caused by such pathogens a challenge for physicians. Taking into account the clinical severity that may be associated with CAP caused by Gram-negative bacteria (respiratory failure, bacteremia, shock, acute respiratory distress syndrome [ARDS]) the magnitude of the global health problem is tremendous.

\footnotetext{
* Correspondence: atorres@clinic.cat

${ }^{1}$ Department of Pneumology, Hospital Clinic of Barcelona, Barcelona, Spain

${ }^{2}$ August Pi i Sunyer Biomedical Research Institute (IDIBAPS), Barcelona, Spain Full list of author information is available at the end of the article
}

We are currently living through an antibiotic resistance crisis, mainly because antibiotics tend to lose their efficacy over time due to the emergence and dissemination of resistance among bacterial pathogens, principally caused by the overuse and inappropriate use of antibiotics, as well as the extensive use of antibiotics in agriculture and the food industry. The Global Point Prevalence Survey (Global-PPS), an international network set up to measure antimicrobial prescription and resistance in the hospital setting, recently published its findings [5]. Pneumonia was the most common illness to receive antibiotic therapy worldwide, accounting for $19 \%$ of patients treated. The most frequently prescribed antibiotics for community-acquired infections were penicillins with a $\beta$-lactamase inhibitor (29\%); amoxicillin with a $\beta$-lactamase inhibitor accounted for $16 \%$ and piperacillin with a $\beta$-lactamase inhibitor accounted for $8 \%$. Third-generation cephalosporins were the second most commonly prescribed antibiotics for community-acquired infections (mainly ceftriaxone, 16\%), followed by fluoroquinolones (14\%).

Antibiotic resistance is a natural phenomenon in bacteria that cannot be stopped; however various measures can be taken in order to reduce the rate of its development and devise more effective strategies to control its spread [6].

Because CAP caused by MDR Gram-negative bacteria is an important clinical concern, we review the main findings concerning the epidemiology, diagnosis and clinical impact of CAP.

\section{CAP caused by Pseudomonas aeruginosa}

The pathogen: Why is it difficult to treat?

$P$. aeruginosa is an opportunistic Gram-negative, non-fermentative bacterium that inhabits the soil and surfaces in aqueous environments. Its high intrinsic antibiotic resistance, broad metabolic versatility and adaptability make it especially difficult to treat. Several studies have shown that the physical characteristics (phenotype) of $P$. aeruginosa isolates vary between those derived from chronic infections, such as cystic fibrosis, and 
those from acute infections, such as pneumonia [7]. Common chromosomal mutations in the mucA gene can convert a non-mucous phenotype into a mucous phenotype. The adaptation of $P$. aeruginosa, which includes complex physiological changes, confers a selective advantage since it can better survive in different habitats [8].

$P$. aeruginosa has intrinsic, adaptive and acquired mechanisms of resistance, the main ones including the presence of $\beta$-lactamases, alterations in membrane permeability due to the presence of ejection pumps, and mutations of transmembrane porins. Furthermore, the capacity to form biofilms (intricate, highly organized bacterial communities, embedded in a matrix composed of exopolysaccharides, DNA and proteins that is attached to a surface and hinders antimicrobial action) favors the persistence of $P$. aeruginosa and makes it more difficult to treat, due to the inherent protection that biofilms provide [8] (Table 1).

A large number of intrinsic $P$. aeruginosa virulence factors are required to establish infection [7]. Moreover, the differential presence or expression of some of these

Table 1 Main resistance mechanisms in Gram-negative pathogens

\begin{tabular}{|c|c|}
\hline Microorganism & Mechanism \\
\hline \multirow[t]{7}{*}{ Pseudomonas aeruginosa } & Efflux pumps \\
\hline & Mutant topoisomerases \\
\hline & Modifying enzymes \\
\hline & AmpC/porin reduction combinations \\
\hline & Metallo-b-lactamases \\
\hline & Outer membrane permeability \\
\hline & Extended spectrum $\beta$-lactamases \\
\hline \multirow[t]{5}{*}{ Acinetobacter baumannii } & $\beta$-lactamases \\
\hline & Efflux pumps \\
\hline & Membrane permeability \\
\hline & Aminoglycoside-modifying enzymes \\
\hline & Alteration of target sites \\
\hline \multirow[t]{5}{*}{ Klebsiella pneumoniae } & Modifying enzyme \\
\hline & Efflux pumps \\
\hline & Mutant topoisomerases \\
\hline & Extended spectrum $\beta$-lactamases \\
\hline & Carbapenemases \\
\hline \multirow[t]{5}{*}{ Stenotrophomonas maltophilia } & $\beta$-lactamases (L1 and L2) \\
\hline & Multidrug efflux pumps \\
\hline & Antibiotic-modifying enzymes \\
\hline & $\begin{array}{l}\text { Mutations of bacterial topoisomerase } \\
\text { and gyrase genes }\end{array}$ \\
\hline & $\begin{array}{l}\text { Reduction in outer membrane } \\
\text { permeability }\end{array}$ \\
\hline
\end{tabular}

virulence factors may determine major inter-strain variability in virulence and thus potentially have a major impact on disease severity and mortality [8].

In conclusion, the pathogenesis of $P$. aeruginosa CAP is very complex, in addition to the broad antimicrobial resistance that limits antibiotic therapy; the virulence of $P$. aeruginosa is certainly a major driver of pneumonia severity and outcome, as well as the different phenotypes described. The capacity to form biofilms provides the bacteria with a further possibility to escape the effects of antibiotics, turning it into a superbug.

\section{Prevalence: What is the prevalence?}

The reported prevalence of CAP caused by $P$. aeruginosa is controversial, largely due to data being limited to single-center studies and because of differences in the study populations [9] (Table 2). Recently, a multinational point-prevalence study analyzed data from 3193 CAP patients in 222 hospitals in 54 countries [10]. The study showed a low prevalence of CAP caused by $P$. aeruginosa (4.2\%), which corresponded to only $11.3 \%$ of patients with culture-positive pneumonia. The prevalences of antibiotic-resistant and MDR P. aeruginosa were also low ( 2 and $1 \%$ respectively). Interestingly, the study reported the prevalence of $P$. aeruginosa in CAP in different continents: $3.8 \%$ in Europe, $4.3 \%$ in North America, 5.2\% in Asia, 4.9\% in South America, 5.5\% in Africa and $3.1 \%$ in Oceania. The prevalence of antibiotic-resistant P. aeruginosa in CAP was $1.6 \%$ in Europe, $2.5 \%$ in North America, 2.2\% in Asia, 3.0\% in South America, and 3.9\% in Africa; there were no reported cases of $P$. aeruginosa antibiotic resistance in Oceania. Finally, the prevalence of MDR P. aeruginosa in CAP was $0.9 \%$ in Europe, $1.2 \%$ in North America, $0.5 \%$ in Asia, $2 \%$ in South America, and $2.3 \%$ in Africa; there were no cases of MDR P. aeruginosa in Oceania.

Similarly, a Spanish study of clinical outcomes and risk factors for CAP caused by MDR and non-MDR $P$. aeruginosa reported a prevalence of MDR $P$. aeruginosa of $1.1 \%$ in a prospective cohort study of 2023 culture-positive CAP patients [11]. The authors also reported that $P$. aeruginosa was an individual risk factor associated with mortality in the study population.

A study by Ferrer et al. [12] of 664 severe CAP cases requiring mechanical ventilation showed that 336 patients had a final microbiological diagnosis; $P$. aeruginosa was reported in $7 \%(n=25)$ of cases $(4 \%$ in the non-invasive mechanical ventilation group and $5 \%$ in the invasive mechanical ventilation group).

\section{$P$. aeruginosa risk factors}

Antibiotic therapy for $P$. aeruginosa is totally different from the standard antimicrobial therapy used to treat common CAP pathogens. Current recommendations 
Table 2 Incidence and risk factors for Pseudomonas aeruginosa community-acquired pneumonia (CAP)

\begin{tabular}{|c|c|c|c|}
\hline Study/year & Population/Country & Prevalence of $P$. aeruginosa & Risk factors for $P$. aeruginosa CAP \\
\hline Aliberti et al. 2013 [2] & $\begin{array}{l}\text { Prospective study of two cohorts } \\
\text { (Barcelona and Edinburgh) } n=1591 \\
\text { CAP patients }\end{array}$ & $\begin{array}{l}\text { Barcelona: } 6.5 \% \text { ( } 32 \text { cases) of these } 12 \\
\text { cases (38\%) were MDR P. aeruginosa } \\
\text { Edinburgh: } 1.6 \% \text { (9 cases) of these } 3 \\
\text { cases (30\%) were MDR P. aeruginosa }\end{array}$ & $\begin{array}{l}\text { Nursing home; hospitalization in the } \\
\text { previous } 90 \text { days; history of chronic } \\
\text { lung disease }\end{array}$ \\
\hline Shindo et al. 2013 [51] & $\begin{array}{l}\text { Prospective study of CAP and HCAP } \\
\text { cases from Japan } n=1431\end{array}$ & $\begin{array}{l}\text { CAP } 3.7 \% \text { (33 cases) HCAP } 8.7 \% \text { ( } 46 \\
\text { cases) }\end{array}$ & \\
\hline Prina et al. 2015 [50] & $\begin{array}{l}\text { Prospective study of CAP from Spain } \\
n=1597\end{array}$ & $4.5 \%$ P. aeruginosa & $\begin{array}{l}\text { Previous antibiotic use, chronic } \\
\text { respiratory diseases, } \mathrm{PO}_{2} / \mathrm{FiO}_{2} \text { ratio } \\
<200\end{array}$ \\
\hline Cillóniz et al. 2016 [11] & $\begin{array}{l}\text { Prospective study of adult patients } \\
\text { with CAP with definitive etiology } \\
n=2023\end{array}$ & $\begin{array}{l}4 \% \text { P. aeruginosa } 1.08 \% \text { MDR } \\
\text { P. aeruginosa }\end{array}$ & $\begin{array}{l}\text { Male sex, chronic respiratory diseases, } \\
\text { C-reactive protein }<12.35 \mathrm{mg} / \mathrm{dL}, \mathrm{PSI} \\
\text { IV to V Prior antibiotic treatment risk } \\
\text { factor for MDR P. aeruginosa }\end{array}$ \\
\hline Restrepo et al. 2018 [10] & $\begin{array}{l}\text { Multicenter, point-prevalence study } \\
\text { of CAP patients ( } 22 \text { hospitals in } 54 \\
\text { countries) } n=3193\end{array}$ & $\begin{array}{l}4.2 \% \text { of all population } 11.3 \% \text { of cases } \\
\text { with defined etiology } 2 \% \text { antibiotic- } \\
\text { resistant } P \text {. aeruginosa } 1 \% \text { MDR } \\
P \text {. aeruginosa }\end{array}$ & $\begin{array}{l}\text { Prior pseudomonas infection/ } \\
\text { colonization, prior tracheostomy, } \\
\text { bronchiectasis, IRVS, very severe COPD }\end{array}$ \\
\hline
\end{tabular}

HCAP healthcare-associated pneumonia, IRVS intensive respiratory or vasopressor support, PSI pneumonia severity index, COPD: chronic obstructive pulmonary disease

provided by international guidelines stratify therapy on the basis of Pseudomonas risk factors [1, 13].

Risk factors for $P$. aeruginosa include structural lung disease (bronchiectasis, chronic obstructive pulmonary disease $[\mathrm{COPD}])$, nursing home residence, C-reactive protein $(\mathrm{CRP})<12.35 \mathrm{mg} / \mathrm{dL}$, prior use of oral steroids, antibiotic therapy within the previous 90 days, and malnutrition $[1,11]$. Chronic $P$. aeruginosa colonization in patients with bronchiectasis and COPD can be an important preliminary step to pneumonia. Non-cystic fibrosis bronchiectasis and COPD account for $2-55 \%$ and for $20-46 \%$ of CAP cases, respectively. These two structural lung conditions facilitate chronic colonization by $P$. aeruginosa, mainly due to failure to eradicate the bacterium during acute infections. As mentioned earlier, $P$. aeruginosa can transform from a non-mucous phenotype to a mucous phenotype which can then adapt to the lung environment and grow as a biofilm.

Interestingly, a recent case-control study [14] identified risk factors for pneumonia due to $P$. aeruginosa susceptible to all routinely tested antipseudomonal $\beta$-lactams (APBL-S) and resistant to at least one antipseudomonal $\beta$-lactam (APBL-R). The authors identified bronchiectasis, interstitial lung disease, prior airway colonization with $P$. aeruginosa and recent exposure to an antipseudomonal $\beta$-lactam as risk factors for APBL-S $P$. aeruginosa in adults with acute bacterial pneumonia.

In the last few years, cases of $P$. aeruginosa infection in previously healthy individuals have been reported; in the majority, heavy exposure to aerosols of contaminated water has been identified [15].

$P$. aeruginosa can establish polymicrobial interactions, mainly with Staphylococcus aureus and Candida albicans that may affect disease management. The relationship between $S$. aureus and $P$. aeruginosa is competitive in nature. The association of $C$. albicans with $P$. aeruginosa or $S$. aureus enhances disease severity in several ways. In vivo studies in rats have shown that pre-colonization of lung tissue by $C$. albicans increases the rate of pneumonia caused by $P$. aeruginosa. This microbial interaction should be taken into account in patients with COPD or non-cystic fibrosis bronchiectasis who are frequently colonized with $P$. aeruginosa and $S$. aureus [16].

According to a recent study, prior antibiotic treatment is a risk factor for $P$. aeruginosa, especially for MDR $P$. aeruginosa [11]. It has been demonstrated that low levels of antibiotics contribute to strain diversification in $P$. aeruginosa; sub-inhibitory and sub-therapeutic antibiotic concentrations induce alterations that effect changes in gene expression, horizontal gene transfer and mutagenesis, which can promote and spread antibiotic resistance.

Given that $P$. aeruginosa CAP has been related to poor clinical outcomes, largely because of inappropriate empiric antibiotic treatment, it is recommended to use empiric anti-pseudomonal cover in all cases of highly suspected MDR $P$. aeruginosa CAP. Risk stratification should take into account the local ecology (prevalence of the pathogen in a specific area) and the patient's risk factors, especially in cases of severe CAP that are associated with higher mortality rates $(20-50 \%)[1]$.

\section{CAP caused by Acinetobacter baumanii}

\section{The pathogen: How important is A. baumanii in CAP?}

A. baumannii is an aerobic Gram-negative coccobacillus. In 2017, the World Health Organization (WHO) included it in the list of "Priority Pathogens", a group of bacteria that poses the greatest threat to human health and for which new antibiotics are urgently needed [17]. 
In recent years, isolates of $A$. baumannii have been recovered from multiple extra-hospital sources, such as vegetables, water treatment plants, fish and shrimp farms, apart from its known natural habitat (soil and humid environments). This broad source of the bacteria may explain the occurrence of community-acquired infections [18].

A. baumannii has a natural resistance to desiccation due to morphological changes that justify its viability for months. Infections caused by A. baumannii may be very difficult to treat due to the ability of the bacterium to evade the host immunity and to form biofilm. Furthermore, A. baumannii has several resistance mechanisms, including $\beta$-lactamases, multidrug efflux pumps, aminoglycoside-modifying enzymes, permeability defects and modifications of target sites [19] (Table 1).

The majority of $A$. baumannii isolates from CAP have a lower antibiotic resistance than isolates from nosocomial infections. Despite this lower rate of antibiotic resistance, severe CAP is the most frequent clinical presentation of $A$. baumannii pneumonia. Caution should be taken when choosing the empirical antibiotic therapy. Although use of a $\$$-lactam plus a macrolide or a fluoroquinolone is the current international guideline recommendation for severe CAP [1], these drug combinations do not completely cover $A$. baumannii, mostly because of its frequent resistance to ceftriaxone [20].

Several studies have reported isolates of MDR A. baumannii in community-dwelling patients and in nursing-home patients. Recently, a prospective cohort study of 651 newly admitted patients in six nursing facilities in the USA reported that $95 \%$ of patients were admitted for post-acute care; $57 \%$ of patients were colonized with MDR pathogens at enrollment. Prolonged hospitalization ( $>14$ days), functional disability, antibiotic use, or device use were the main risk factors for colonization at enrollment. The rate per 1000 patient-days of acquiring a new resistant Gram-negative bacillus was 13.6\%. MDR colonization at discharge for resistant Gram-negative bacilli was 34\% [21].

In a study from west China [22], investigating the antimicrobial susceptibility of 32 isolates of $A$. baumannii causing CAP, the authors reported that $30 \%$ of the isolates were resistant to the majority of the antibiotics; the resistant rates to imipenem and meropenem were 19 and $9 \%$, respectively. An important finding of this study is that approximately $80 \%$ of the patients had had a previous hospital admission.

An MDR A. baumannii was recently isolated in a previously healthy patient with CAP from Hong Kong. The patient was treated with intravenous colistin and oral monocycline and recovered fully [23]. This case is not isolated and more and more reports of cases of CAP caused by MDR A. baumannii are being published.
In conclusion, although $A$. baumannii is not a frequent cause of CAP, the capacity to rapidly develop resistance mechanisms to antibiotics and the fulminant clinical presentation (with a mortality rate around 50\%) make this pathogen an important health problem, especially in tropical and subtropical areas.

\section{Prevalence: What is the prevalence?}

During the last 10 years, A. baumannii has been described as a rare cause of CAP but with clinical relevance. The great majority of CAP cases caused by $A$. baumannii occurs in countries with tropical or sub-tropical climates. A. baumannii is an emerging pathogen in the regions of Asia-Pacific, with a higher prevalence in Hong Kong, Singapore, Taiwan, South Korea and Australia [24, 25].

CAP cases caused by A. baumannii are very rare in Europe and USA. A recent USA case report of severe CAP and review of the literature found that 19 cases of CAP caused by $A$. baumannii have been reported in the USA to date [26].

Unlike nosocomial pneumonia caused by A. baumannii, CAP cases caused by A. baumannii have a seasonal presentation, with the highest prevalence during the warm and humid months of the year [25].

\section{A. baumannii risk factors}

The clinical presentation of A. baumannii CAP is fulminant with acute onset of fever, dyspnea and rapid progression to respiratory failure and shock. A. baumannii CAP is associated with a mortality rate ranging from 40 to $60 \%$ especially in cases presenting with bacteremia and shock. The main risk factors related to this infection are alcoholism, diabetes mellitus and chronic lung disease [24].

It is known that alcohol abuse impacts on the innate and adaptive immune response. A study published in 2013 described the impact of alcohol on macrophage-like cell antimicrobial functions in A. baumannii infections [27]. The authors demonstrated that alcohol plays an important role in inhibiting nitric oxide $(\mathrm{NO})$ production which is essential to eradicate bacteria exposed to macrophages after phagocytosis. Alteration of intra- and extra-cellular NO production promotes microbial survival, facilitating intracellular replication. Furthermore, macrophages exposed to alcohol have lower production of pro-inflammatory tumor necrosis factor (TNF)- $\alpha$, interleukin (IL)-1 $\beta$, and IL-12 and increased levels of IL- 6 . This imbalance in the production of cytokines affects the differentiation of naïve $\mathrm{T}$ cells into Th1 cells because of low IL-12 production, and the production of interferon (IFN) $-\gamma$ from $\mathrm{T}$ and natural killer (NK) cells. Moreover, it is known that low levels of TNF- $\alpha$ and IL-1 $\beta$ are 
associated with septic shock and the risk of bacterial infections [27].

A related study recently published by Kamoshida et al. [28] described for the first time the ability of $A$. baumannii to inhibit the formation of neutrophil extracellular traps (NETs), thus suppressing neutrophil adhesion. It is well known that the main role of NETs is to prevent microbial dissemination and to eradicate pathogens. The ability of $A$. baumannii to escape the immune response may explain the fulminant clinical presentation of CAP caused by this pathogen.

An Australian study suggested that microaspiration of pharyngeal $A$. baumannii may be responsible for CAP in alcoholic patients [29]. In this study, $10 \%$ of community residents attending the Emergency Department in the wet-season (March-April) with non-respiratory diseases, no episode of previous hospitalization and a history of alcohol abuse (alcohol intake $>6$ standard drinks/day) had A. baumannii in their throat [29].

The susceptibility to pneumonia in patients with diabetes can be ascribed to several factors. Patients with diabetes have an increased risk of hyperglycemia (which reduces the mobilization of polymorphonuclear leukocytes, chemotaxis and phagocytic activity), increased risk of aspiration, impaired immunological defenses, and deterioration in lung function and chronic complications, such as neuropathy [30].

Patients with underlying chronic lung disease, such as COPD or bronchiectasis, also show reduced innate defense mechanisms. Generally, these patients are smokers, passive smokers or ex-smokers and the frequent use of inhaled corticosteroids make them more vulnerable to infections such as pneumonia [10, 11, 27].

In conclusion, because of its possible fulminating course, CAP caused by A. baumannii should be suspected in patients with specific risk factors and a severe presentation of pneumonia. Early recognition and prompt initiation of broad empirical antibiotic coverage are mandatory to limit the high mortality related to this infection.

\section{CAP caused by Klebsiella pneumoniae}

The pathogen: What is so special about $K$. pneumoniae in CAP?

K. pneumoniae is a Gram-negative capsulate bacterium responsible for severe pneumonia especially in immunocompromised patients. In the last two decades, there has been an increase in antibiotic resistance in $K$. pneumoniae isolates globally. The emergency of MDR and hypervirulent $K$. pneumoniae strains has been reported in immunocompromised patients and in healthy persons [31].

The main mechanisms of antibiotic resistance in $K$. pneumoniae are the expression of extended spectrum $\beta$-lactamases (ESBLs), which confers resistance against penicillins, cephalosprins and monobactams, and the expression of carbapenemases, which confers resistance against all $\beta$-lactams including carbapenems (Table 1).

Interestingly, hypervirulent $K$. pneumoniae strains produce a hypercapsule, also known as being hypermucoviscous. The capsule, a polysaccharide matrix that coats the cell, is necessary for K. pneumoniae virulence and is arguably the most thoroughly studied virulence factor of K. pneumoniae [31]. Although antimicrobial resistance in hypervirulent $K$. pneumoniae is generally lower than in non-hypervirulent $K$. pneumoniae strains, cases with more resistant strains of hypervirulent $K$. pneumoniae have recently been reported [32].

K. pneumoniae has the ability to avoid phagocytosis. A recent experimental study found that the capsule is dispensable for intracellular Klebsiella survival if bacteria are not opsonized. K. pneumoniae survives the killing by macrophages by manipulating phagosome maturation, which may contribute to Klebsiella pathogenesis [33].

In conclusion, CAP caused by MDR and hypervirulent $K$. pneumoniae represents a great challenge in terms of treatment and management, especially in Asian countries, where the majority of cases are reported.

\section{Prevalence: What is the prevalence?}

A recent 5-year study in a French ICU [34] described 59 infections caused by $K$. pneumoniae; 26 (44\%) of them were community-onset infections. Interestingly, the authors reported 12 hypervirulent $K$. pneumoniae strains in the group of community-onset infections, $6(50 \%)$ of which were isolated from patients with CAP. The authors observed that hypervirulent $K$. pneumoniae cases had higher rates of organ failure compared with non-hypervirulent cases. However, mortality rates were similar in the two groups.

In a study performed in Cambodia [35], 2315 patients with acute lower respiratory infections were enrolled, including 587 (25\%) whose bacterial etiology could be assigned. K. pneumoniae was identified in $8 \%(n=47)$ of the microbiologically-confirmed cases. ESBL-producing strains were found in $8(17 \%)$ patients. The main risk factors related to K. pneumoniae infection were female sex and diabetes mellitus. The overall mortality rate due to $K$. pneumoniae infection was $38 \%$.

Approximately $1-7 \%$ of cases of CAP are caused by $K$. pneumoniae, with $5-36 \%$ of these being MDR strains [2, 36]. Interestingly, in Asian countries (Taiwan, Cambodia, Shanghai) $K$. pneumoniae is described as a frequent pathogen causing bacteremia [37, 38]. Although reports of cases from Europe and USA are generally rare, an increasing incidence from these regions has been registered in recent years [34, 39]. 


\section{K. pneumoniae risk factors}

The recognized risk factors for $K$. pneumoniae CAP are female sex, diabetes mellitus and alcoholism. MDR $K$. pneumoniae can be recognized as a virulent pathogen causing severe CAP, frequently associated with septic shock, respiratory failure and bacteremia. Morbidity and mortality rates are high especially in Asian countries were the pathogen is reported in younger patients with no chronic conditions. This pattern may be explained by the virulence of the strains (especially hypervirulent $K$. pneumoniae) and the high rate of antibiotic consumption in these regions $[35,40]$.

\section{CAP caused by Stenotrophomonas maltophilia} The pathogen: What is so special about S. maltophilia in CAP?

S. maltophilia is a motile, aerobic, non-fermentative Gram-negative bacillus with the ability to survive on any humid surface, form biofilm and colonize humid surfaces. It is an emerging opportunistic pathogen with multidrug resistance, which particularly affects immunocompromised patients (i.e., with malignances, post-organ transplantation) worldwide [41, 42].

S. maltophilia is intrinsically resistant to carbapenems and frequently carries genetic elements that provide resistance to other $\beta$-lactams, fluoroquinolones, aminoglycosides and tetracyclines. S. maltophilia has the ability to acquire genes involved in antibiotic resistance from other bacterial species. The most relevant mechanisms of antibiotic resistance include $\beta$-lactamase (L1 and L2) production, multidrug efflux pumps (which confer resistance to macrolides, quinolones, aminoglucosydes, polymyxins), antibiotic-modifying enzymes, mutations of bacterial topoisomerase and gyrase genes and reduction in outer membrane permeability [42] (Table 1).

Although S. maltophilia has been reported in patients with cystic fibrosis, it can affect healthy individuals through contaminated wounds or infected catheters [43, 44]. Transmission of S. maltophilia may occur through direct contact with contaminated source such as contaminated water or medical devices [42].

In conclusion, CAP caused by $S$. maltophilia is associated with high mortality rates. Hemorrhagic pneumonia is one of the most severe forms of S. maltophilia infection [45]. It is associated with poor outcome despite appropriate antibiotic therapy.

\section{Prevalence: What is the prevalence?}

There is limited information on the worldwide prevalence of community-acquired S. maltophilia pneumonia. The majority of data comes from case reports [46]. $S$. maltophilia has been reported as an important cause of CAP in patients with hematologic diseases, with a prevalence of bacteremia ranging between 2 and 7\%. The mortality rate is approximately 35\% (ranging between 30 and $40 \%)$ in this special population.

\section{S. maltophilia risk factors}

The majority of patients affected by this pathogen have previous chronic comorbidities, such as COPD, cystic fibrosis, malignancy (especially hematologic diseases), human immunodeficiency virus (HIV)-infection (acquired immunodeficiency syndrome [AIDS]) or other immunodeficiencies. Patients with prior antimicrobial therapy, prolonged hospitalization, indwelling catheters, mechanically ventilated and receiving corticosteroids have an increased risk of $S$. maltophilia CAP $[42,46]$.

\section{Diagnosis: Is it possible to predict gram-negative MDR pathogens in CAP?}

The reference microbiological diagnostic tools for bacteria causing respiratory tract infections remain the Gram stain and semi-quantitative conventional cultures from direct respiratory samples. Bacterial identification is currently based on matrix-assisted latex laser desorption/ionization time-of-flight mass spectrometry (MALDI-TOF MS) and susceptibility testing. As a consequence of the time needed for microbiological diagnosis, many patients initially receive inappropriate antibiotic treatment, which may increase morbidity and mortality.

Molecular techniques based on multiplex PCR have also been developed in recent years in order to simultaneously identify and quantify multiple respiratory pathogens from different types of samples in a single procedure [47]. However, the main challenge for the rapid diagnosis of respiratory infections is the early detection of the antibiotic resistance profile of the various bacteria. The biggest obstacle in the use of molecular techniques for detecting resistance is the discrepancy between genotype and phenotype, the continuous discovery of new resistance mechanisms and, as a result, the potential presence of unknown mechanisms, which may lead to false negative results using molecular techniques [47].

Recognizing patients at risk of colonization with MDR Gram-negative bacteria, such as patients with bronchiectasis or COPD (frequently colonized by P. aeruginosa), is essential, since several studies have reported the association between previous colonization and risk of pneumonia $[48,49]$.

Currently, there is no specific score to predict MDR Gram-negative pathogens in CAP. However, several scoring systems have been proposed to identify patients with risk factors for developing CAP caused by MDR pathogens. The Aliberti score [2] takes into account different variables and gives a specific score to each of them: chronic renal failure (5 points), prior hospitalization (4 
points), nursing home residence (3 points) and other variables ( 0.5 points each). CAP patients with an Aliberti score $\geq 3$ points have a reported prevalence of MDR of $38 \%$, whereas CAP patients with an Aliberti score of $\leq 0.5$ have a reported prevalence of MDR of $8 \%$. The PES ( $P$. aeruginosa, Enterobacteriaceae ESBL-positive, methicillinresistant $S$. aureus [MRSA]) score includes 1 point each for age 40-65 years and male sex; 2 points each for age > 65 years, previous antibiotic use, chronic respiratory disorder, and impaired consciousness; 3 points for chronic renal failure; and minus 1 point if fever is present initially. The risk of MDR pathogens is higher with 5 points or more [50]. Unfortunately, studies validating the Aliberti and PES score do not include immunocompromised patients. The ability of these scores to identify risk factors for CAP by MDR pathogens in this population is still unclear.

An important study by Shindo et al. [51] investigated the main risk factors for MDR pathogens in 1413 patients with CAP. Six risk factors were identified: prior hospitalization, immunosuppression, previous antibiotic use, use of gastric acid-suppressive agents, tube feeding and non-ambulatory status. Unlike the Aliberti and PES studies, Shindo's study included immunocompromised patients. Interestingly, the authors analyzed risk factors for Gram-positive (MRSA) and Gram-negative pathogens separately. The authors observed that the risk of MDR Gram-negative pathogens did not increase in the presence of $\geq 3$ risk factors; conversely, for Gram-positive (MRSA) pathogens, the risk increased in the presence of $\geq 2$ risk factors, especially if one of the risk factors was specific for MRSA, such as previous colonization or previous hospitalization.

\section{Therapy: How is it possible to treat these pathogens?}

Since antibiotic treatment for $P$. aeruginosa is completely different from standard therapy to cover the most common pathogens in CAP, current international guidelines for severe CAP stratify therapy recommendations on the basis of $P$. aeruginosa risk factors [1]. MDR $P$. aeruginosa should be covered only in cases that are strongly suspected because of concurrent risk factors. It is important to underline that prior antibiotic therapy has been reported as the only risk factor for MDR $P$. aeruginosa in CAP patients [11].

Empirical antibiotic treatment based on the evaluation of the patient's risk factors is also of pivotal importance in the management of A. baumannii CAP.

Because CAP caused by $A$. baumannii has a fulminant clinical presentation with a high mortality rate (approximately $50 \%$ ), greater attention should be paid in elderly patients with multi-comorbidities, and patients with alcohol abuse, chronic lung disease and prior antibiotic therapy, especially in Asian countries where this pathogen is frequently reported. Early appropriate antimicrobial therapy is critical. Recommended empirical antibiotic therapy for A. baumannii CAP includes anti-pseudomonal penicillins, aminoglycoside, ciprofloxacin, and imipenem. Tigecycline, colistin, ceftazidime, doxycycline, minocycline, piperacillin/tazobactam, tobramycin, rifampin, fosfomycin and levofloxacin are active against variable percentages of strains. The treatment should include an association of two or more of these antibiotics according to the antibiogram of the isolated pathogen. Moreover some countries, notably Asia [52] and Australia [53], have implemented specific antibiotic recommendations in cases of severe CAP in order to cover pathogens such as A. baumannii.

In CAP caused by $K$. pneumoniae, depending on the clinical severity and the risk of infection by a strain with resistance mechanisms (production of ESBLs, cefaminases or carbapenemases), a 3rd generation cephalosporin (cefotaxime or ceftriaxone) or a fluoroquinolone (ciprofloxacin or levofloxacin) should be used. Another possibility, in case of infection by carbapenemase-producing or carbapenem-resistant strains (due to loss of porin and hyperproduction of Amp-C), is the use of associations of two or three of the following antibiotics: colistin, tigecycline, fosfomycin (if the strain is sensitive), an aminoglycoside (amikacin or gentamicin) and meropenem (if the minimum inhibitory concentration $[\mathrm{MIC}]=16 \mathrm{mg} / \mathrm{L}$ ), administered in high doses and by continuous perfusion. Hypervirulent strains (serotypes K1 and K2) are usually more sensitive to antibiotics, but production of K. pneumoniae-type carbapenemase has also been observed.

For the antibiotic treatment of S. maltophilia pneumonia, trimethoprim-sulfamethoxazole (TMP-SMX) is still considered the drug of choice despite increasing resistance. It is preferably used in association with another antibiotic depending on the severity of the pneumonia and the sensitivity of the pathogen. Other alternative antibiotics include $\beta$-lactams (i.e., ceftazidime), fluoroquinolones (i.e., levofloxacin, moxifloxacin), minocycline or tigecycline. In vitro, the following associations are often synergistic: cotrimoxazole with colistin, tigecycline, ceftazidime and rifampicin; colistin with tigecycline, ceftazidime and rifampin; ceftazidime with levofloxacin, moxifloxacin and aztreonam.

Figure 1 proposes an algorithm for the empiric antibiotic therapy of CAP in patients with risks factors for MDR pathogens.

\section{Conclusion}

Correct identification of CAP patients suspected of being infected with MDR Gram-negative pathogens is crucial. Specific risk factors, local ecology and resistance patterns should always be considered in order to 


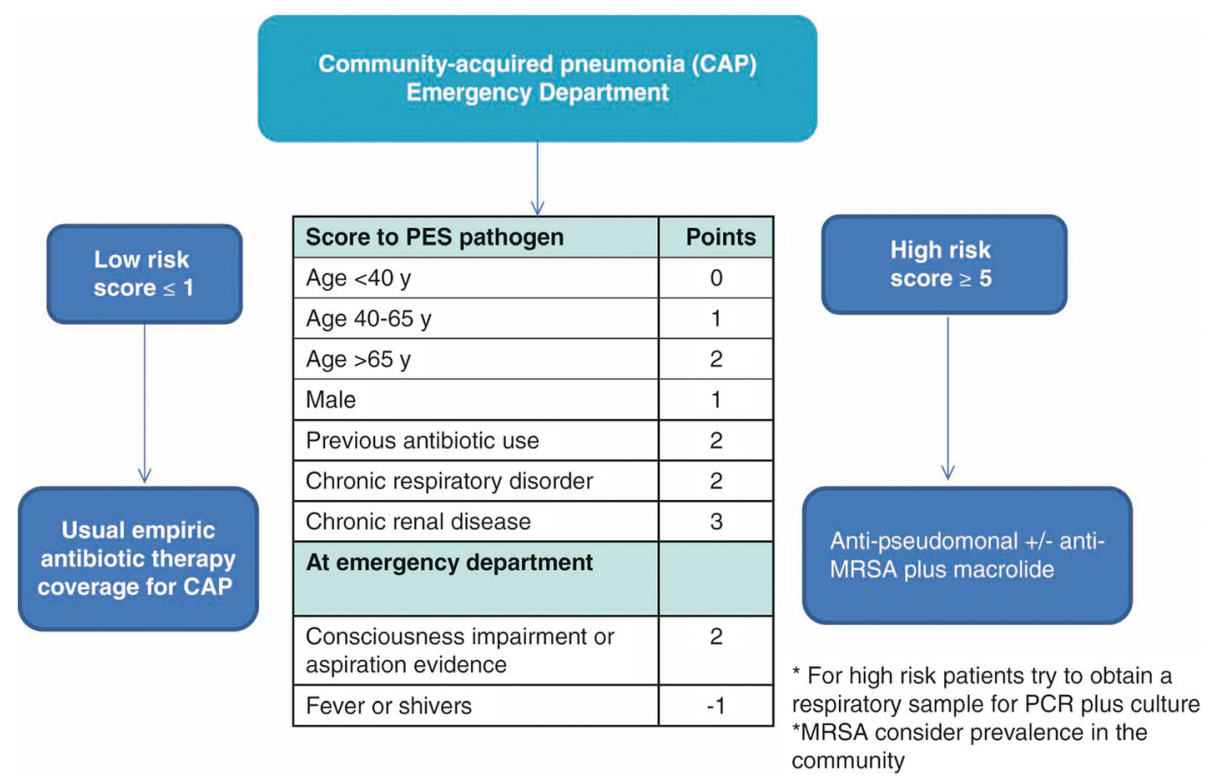

Fig. 1 Proposed algorithm for empiric antibiotic therapy in community-acquired pneumonia for patients with risk of multidrug resistant (MDR) pathogens using the PES (Pseudomonas aeruginosa, Enterobacteriaceae extended-spectrum $\beta$-lactamase-positive, methicillin-resistant Staphylococcus aureus [MRSA]) score from [50]. PCR polymerase chain reaction

determine the adequate empirical antibiotic therapy. Early hemodynamic and respiratory support is critical in these patients since the majority of cases of pneumonia may become fulminant systemic disease with both respiratory failure and multiple organ dysfunction.

The collaboration of a multidisciplinary team (critical care specialists, pneumologist, infectious disease specialists, microbiologists) improves management of the most severe cases. The role of the microbiology laboratory, in particular, is of pivotal importance to determine the antimicrobial susceptibility pattern of the pathogen causing CAP, so that appropriate antibiotic therapy can be initiated as soon as possible, avoiding excessive use of broad spectrum antimicrobials, which increase the selection of resistant pathogens.

\section{Acknowledgements}

Dr. Cillóniz is the recipient of a Postdoctoral Grant (Strategic plan for research and innovation in health-PERIS 2016-2020).

\section{Funding}

Dr. Cillóniz is the recipient of a Postdoctoral Grant (Strategic plan for research and innovation in health-PERIS 2016-2020). Publication cost were funded by Ciber de Enfermedades Respiratorias (CibeRes CB06/06/0028), 2009 Support to Research Groups of Catalonia 911, IDIBAPS (CERCA

Programme / Generalitat de Catalunya).

\section{Availability of data and materials}

Not applicable.

\section{Authors' contributions}

$C C, C D$ and AT conceived, provided expertise, wrote the raft and contributed to revising the manuscript. All authors read and approved the final manuscript.

Ethics approval and consent to participate Not applicable.

\section{Consent for publication}

Not applicable.

\section{Competing interests}

The authors declare that they have no competing interests.

\section{Publisher's Note}

Springer Nature remains neutral with regard to jurisdictional claims in published maps and institutional affiliations.

\section{Author details}

'Department of Pneumology, Hospital Clinic of Barcelona, Barcelona, Spain. ${ }^{2}$ August Pi i Sunyer Biomedical Research Institute (IDIBAPS), Barcelona, Spain. ${ }^{3}$ University of Barcelona, Barcelona, Spain. ${ }^{4}$ Biomedical Research Networking Centres in Respiratory Diseases (CIBERES), Barcelona, Spain. ${ }^{5}$ Department of Anesthesiology and Intensive Care Medicine, Fondazione Policlinico Universitario A. Gemelli, Università Cattolica del Sacro Cuore, Rome, Italy.

Published online: 09 March 2019

\section{References}

1. Mandell LA, Wunderink RG, Anzueto A, et al. Infectious Diseases Society of America/American Thoracic Society consensus guidelines on the management of community-acquired pneumonia in adults. Clin Infect Dis. 2007;44(Suppl 2):S27-72

2. Aliberti S, Cilloniz C, Chalmers JD, et al. Multidrug-resistant pathogens in hospitalised patients coming from the community with pneumonia: a European perspective. Thorax. 2013;68:997-9.

3. Cillóniz C, Ewig S, Polverino E, et al. Microbial aetiology of communityacquired pneumonia and its relation to severity. Thorax. 2011;66:340-6.

4. Magiorakos A-P, Srinivasan A, Carey RB, et al. Multidrug-resistant, extensively drug-resistant and pandrug-resistant bacteria: an international expert proposal for interim standard definitions for acquired resistance. Clin Microbiol Infect. 2012;18:268-81.

5. Versporten A, Zarb P, Caniaux I, et al. Antimicrobial consumption and resistance in adult hospital inpatients in 53 countries: results of an internetbased global point prevalence survey. Lancet Glob Health. 2018;6:e619-29.

6. European Antimicrobial Resistance Surveillance Network. Surveillance of antimicrobial resistance in Europe 2016. 2016. Available at: https://ecdc. 
europa.eu/sites/portal/files/documents/AMR-surveillance-Europe-2016.pdf. Accessed 19 Nov 2018.

7. Gellatly SL, Hancock REW. Pseudomonas aeruginosa: new insights into pathogenesis and host defenses. Pathog Dis. 2013;67:159-73.

8. Crousilles $\mathrm{A}$, Maunders $\mathrm{E}$, Bartlett $\mathrm{S}$, et al. Which microbial factors really are important in Pseudomonas aeruginosa infections? Future Microbiol. 2015;10:1825-36.

9. von Baum H, Welte T, Marre R, et al. Community-acquired pneumonia through Enterobacteriaceae and Pseudomonas aeruginosa: diagnosis, incidence and predictors. Eur Respir J. 2010;35:598-605.

10. Restrepo MI, Babu BL, Reyes LF, et al. Burden and risk factors for pseudomonas aeruginosa community-acquired pneumonia: a multinational point prevalence study of hospitalised patients. Eur Respir J. 2018;52: 1709910.

11. Cillóniz C, Gabarrús A, Ferrer M, et al. Community-acquired pneumonia due to multidrug and non-multidrug resistant Pseudomonas aeruginosa. Chest. 2016;150:415-25.

12. Ferrer M, Travierso C, Cilloniz C, et al. Severe community-acquired pneumonia: characteristics and prognostic factors in ventilated and nonventilated patients. PLoS One. 2018;13:e0191721.

13. Lim WS, Baudouin SV, George RC, et al. BTS guidelines for the management of community acquired pneumonia in adults: update 2009. Thorax. 2009; 64(Suppl 3):iii -55 .

14. Al-Jaghbeer MJ, Justo JA, Owens W, et al. Risk factors for pneumonia due to beta-lactam-susceptible and beta-lactam-resistant Pseudomonas aeruginosa: a case-case-control study. Infection. 2018. https://doi.org/10.1007/s15010018-1147-z [Epub ahead of print].

15. John TJ, Lalla U, Taljaard JJ, et al. An outbreak of community-acquired pseudomonas aeruginosa pneumonia in a setting of high water stress. QJM. 2017;110:855-6.

16. Cillóniz C, Civljak R, Nicolini A, Torres A. Polymicrobial community-acquired pneumonia: an emerging entity. Respirology. 2016;21:65-75.

17. WHO. High levels of antibiotic resistance found worldwide, new data shows 2018. Available at: WHO. http:/www.who.int/mediacentre/news/releases/ 2018/antibiotic-resistance-found/en/. Accessed 25 Jun 2018.

18. Eveillard M, Kempf M, Belmonte $\mathrm{O}$, et al. Reservoirs of Acinetobacter baumannii outside the hospital and potential involvement in emerging human community-acquired infections. Int J Infect Dis. 2013;17:e802-5.

19. Wong D, Nielsen TB, Bonomo RA, et al. Clinical and pathophysiological overview of acinetobacter infections: a century of challenges. Clin Microbiol Rev. 2017;30:409-47.

20. Dexter C, Murray GL, Paulsen IT, Peleg AY. Community-acquired Acinetobacter baumannii: clinical characteristics, epidemiology and pathogenesis. Expert Rev Anti-Infect Ther. 2015;13:567-73.

21. Mody L, Foxman B, Bradley S, et al. Longitudinal assessment of multidrugresistant organisms in newly admitted nursing facility patients: implications for an evolving population. Clin Infect Dis. 2018;67:837-44.

22. Peng $\mathrm{C}$, Zong Z, Fan H. Acinetobacter baumannii isolates associated with community-acquired pneumonia in West China. Clin Microbiol Infect. 2012; 18:E491-3.

23. Son YW, Jung IY, Ahn MY, et al. A case of community-acquired pneumonia caused by multidrug-resistant Acinetobacter baumannii in Korea. Infect Chemother. 2017;49:297-300.

24. Ong CWM, Lye DCB, Khoo KL, et al. Severe community-acquired Acinetobacter baumannii pneumonia: an emerging highly lethal infectious disease in the Asia-Pacific. Respirology. 2009;14:1200-5.

25. Kim YA, Kim JJ, Won DJ, Lee K. Seasonal and temperature-associated increase in community-onset Acinetobacter baumannii complex colonization or infection. Ann Lab Med. 2018;38:266-70.

26. Serota DP, Sexton ME, Kraft CS, Palacio F. Severe community-acquired pneumonia due to Acinetobacter baumannii in North America: case report and review of the literature. Open Forum Infect Dis. 2018:5:0fy044.

27. Eugenin EA. Community-acquired pneumonia infections by Acinetobacter baumannii: how does alcohol impact the antimicrobial functions of macrophages? Virulence. 2013;4:435-6.

28. Kamoshida G, Kikuchi-Ueda T, Nishida S, et al. Pathogenic bacterium Acinetobacter baumannii inhibits the formation of neutrophil extracellular traps by suppressing neutrophil adhesion. Front Immunol. 2018;9:178.

29. Anstey NM, Currie BJ, Hassell M, Palmer D, Dwyer B, Seifert H. Communityacquired bacteremic Acinetobacter pneumonia in tropical Australia is caused by diverse strains of Acinetobacter baumannii, with carriage in the throat in at-risk groups. J Clin Microbiol. 2002;40:685-6.
30. Peleg AY, Weerarathna T, McCarthy JS, Davis TME. Common infections in diabetes: pathogenesis, management and relationship to glycaemic control. Diabetes Metab Res Rev. 2007;23:3-13.

31. Lee $\mathrm{CR}$, Lee JH, Park KS, et al. Antimicrobial resistance of hypervirulent Klebsiella pneumoniae: epidemiology, hypervirulence-associated determinants, and resistance mechanisms. Front Cell Infect Microbiol. 2017;7:483.

32. Gu D, Dong N, Zheng Z, et al. A fatal outbreak of ST11 carbapenemresistant hypervirulent Klebsiella pneumoniae in a Chinese hospital: a molecular epidemiological study. Lancet Infect Dis. 2018;18:37-46.

33. Cano V, March C, Insua JL, et al. Klebsiella pneumoniae survives within macrophages by avoiding delivery to lysosomes. Cell Microbiol. 2015;17: 1537-60.

34. Rafat C, Messika J, Barnaud G, et al. Hypervirulent Klebsiella pneumoniae, a 5-year study in a French ICU. J Med Microbiol. 2018;67:1083-9.

35. Rammaert B, Goyet S, Beauté J, et al. Klebsiella pneumoniae related community-acquired acute lower respiratory infections in Cambodia: clinical characteristics and treatment. BMC Infect Dis. 2012;12:3.

36. Ishida T, Ito A, Washio Y, et al. Risk factors for drug-resistant pathogens in immunocompetent patients with pneumonia: evaluation of PES pathogens. $J$ Infect Chemother. 2017:23:23-8.

37. Lin $Y T$, Jeng $Y Y$, Chen TL, Fung C-P. Bacteremic community-acquired pneumonia due to Klebsiella pneumoniae: clinical and microbiological characteristics in Taiwan, 2001-2008. BMC Infect Dis. 2010;10:307.

38. Tseng C-P, Wu H-S, Wu T-H, et al. Clinical characteristics and outcome of patients with community-onset Klebsiella pneumoniae bacteremia requiring intensive care. J Microbiol Immunol Infect. 2013:46:217-23.

39. Decré $D$, Verdet $C$, Emirian $A$, et al. Emerging severe and fatal infections due to Klebsiella pneumoniae in two university hospitals in France. J Clin Microbiol. 2011;49:3012-4.

40. Inghammar M, Borand L, Goyet S, et al. Community-acquired pneumonia and gram-negative bacilli in Cambodia-incidence, risk factors and clinical characteristics. Trans R Soc Trop Med Hyg. 2018;112:57-63.

41. Baker TM, Satlin MJ. The growing threat of multidrug-resistant gramnegative infections in patients with hematologic malignancies. Leuk Lymphoma. 2016;57:2245-58.

42. Brooke JS. Stenotrophomonas maltophilia: an emerging global opportunistic pathogen. Clin Microbiol Rev. 2012;25:2-41.

43. Cha YK, Kim JS, Park SY, et al. Computed tomography findings of community-acquired Stenotrophomonas maltophilia pneumonia in an immunocompetent patient: a case report. Korean J Radiol. 2016;17:961-4.

44. Geller M, Nunes CP, Oliveira L, Nigri R. S. maltophilia pneumonia: a case report. Respir Med Case Rep. 2018;24:44-5.

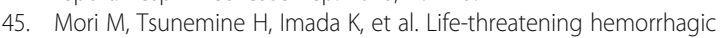
pneumonia caused by Stenotrophomonas maltophilia in the treatment of hematologic diseases. Ann Hematol. 2014;93:901-11.

46. Falagas ME, Kastoris AC, Vouloumanou EK, Dimopoulos G. Communityacquired Stenotrophomonas maltophilia infections: a systematic review. Eur J Clin Microbiol Infect Dis. 2009;28:719-30.

47. Torres A, Lee N, Cilloniz C, et al. Laboratory diagnosis of pneumonia in the molecular age. Eur Respir J. 2016:48:1764-78.

48. Sibila O, Rodrigo-Troyano A, Shindo Y, et al. Multidrug-resistant pathogens in patients with pneumonia coming from the community. Curr Opin Pulm Med. 2016;22:219-26.

49. Gross AE, Van Schooneveld TC, Olsen KM, et al. Epidemiology and predictors of multidrug-resistant community-acquired and health careassociated pneumonia. Antimicrob Agents Chemother. 2014;58:5262-8.

50. Prina E, Ranzani OT, Polverino E, et al. Risk factors associated with potentially antibiotic-resistant pathogens in community-acquired pneumonia. Ann Am Thorac Soc. 2015;12:153-60.

51. Shindo $Y$, Ito R, Kobayashi $D$, et al. Risk factors for drug-resistant pathogens in community-acquired and healthcare-associated pneumonia. Am J Respir Crit Care Med. 2013;188:985-95.

52. Cao B, Huang Y, She DY, et al. Diagnosis and treatment of communityacquired pneumonia in adults: 2016 clinical practice guidelines by the Chinese thoracic society, Chinese Medical Association. Clin Respir J. 2018;12: $1320-60$.

53. National Health and Medical Research Council. Therapeutic guidelines antibiotic version 15. In: Australian Clinical Practice Guidelines. Canberra: National Health and Medical Research Council; 2014. 